\title{
Role Emerging Placements in Physiotherapy: Student Experiences and Practical Considerations
}

\author{
R. Kyte, H. Frank, B. Ransley, K. Wood and Y. Thomas \\ University of Worcester, UK, email: r.kyte@worc.ac.uk
}

\section{Purpose}

Physiotherapists work in increasingly diverse areas of practice across both health and social care sectors. Consequently, education providers are being urged to ensure students graduate with the necessary skills to work flexibly in a range of environments in both traditional and emerging roles (1). Role emerging placements (REPs) - i.e. those conducted in settings where there may be little or no existing therapy provision - are commonly used within occupational therapy (OT) to help prepare students for contemporary practice by providing opportunities to improve their problem-solving skills, leadership and autonomy through development of their own professional role (2).

There is, however, little published literature on the use of REPs in Physiotherapy. This poster will consider the practicalities and student experiences of REPs in Physiotherapy (3).

\begin{abstract}
Methods
Staff collaborated with OT colleagues to develop REPs with local partners who had identified a need for Physiotherapy. REPs were allocated to a selection of $2^{\text {nd }}$ year and $3^{\text {rd }}$ year students. Students were supported on a daily basis by an onsite-supervisor, and a qualified clinician provided off-site or 'long-armed' supervision to help students draw on their knowledge of theory to develop their own role.

Students who had undertaken a REP were invited to take part in a focus group, which used a research informed semi-structured topic guide to explore their experiences (3). Six participants volunteered to take part. Group discussions were digitally recorded, professionally transcribed verbatim and thematically analysed. Ethical approval was gained from the University of Worcester. University staff who had been involved in establishing REPs were asked to share their experiences regarding the practicalities of successfully implementing REPs.
\end{abstract}

\section{Results}

Students successfully completed REPs in a range of settings including; residential and nursing homes, learning disabilities, neurological charities and mental health. Thematic analysis of student focus group data revealed 5 main themes:
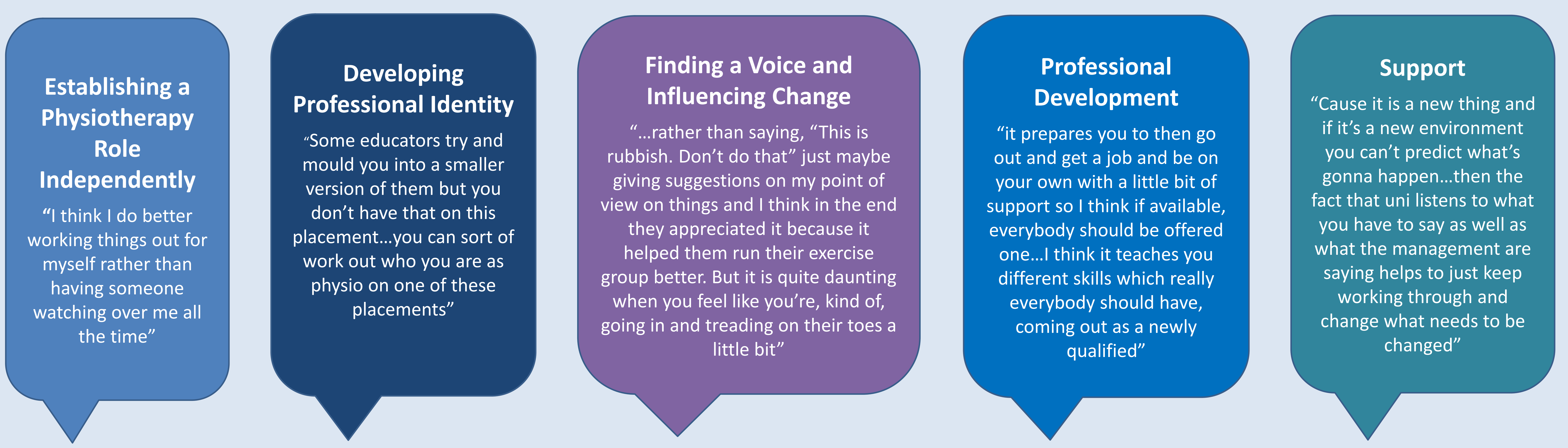

\section{Practical Considerations: University Staff Experiences}

\section{Planning}

- Ensure students \& onsite staff are appropriately briefed prior to starting placement; pre-placement meetings or visits are advisable

- Ensure a structured induction is set up to allow students time to observe the existing organisation/service

\section{Support}

- Establish a suitable communication method between the 'long armed' supervisor, on-site supervisor and students

- Organise supervision dates $\&$ times in advance

- Where possible, pair students to facilitate peer support and learning

\section{Conclusions}

REPs appear to offer a non-traditional developmental learning opportunity where physiotherapy students can develop the skills required to deal with change and adapt to new roles. Students reported both positive and negative experiences, but most students felt the REPs had resulted in a number of professional and personal benefits. Adequate time and resources are required to ensure all parties are prepared and supported.

\section{Logistics on Placement}

- Set clear expectations from the start in terms of communication and scope of practice

- Negotiate the focus of the placement based on the students' previous experiences, strengths, weaknesses, areas of interest and negotiate this with the organisation; give students time to work this out

- Have a clear structure for communication between students and onsite staff for handover \& feedback

- Identify how the students will document their interventions and how/when these will be countersigned

- Ensure appropriate time is built into working hours for research, study and reflection

- Consider access to computers, internet, telephones, study area \& lockers

\section{Implications}

- REPs have the potential to both increase placement capacity and equip students with the skills to deal with a changing healthcare environment. However, it is important to publicize both the challenges and benefits associated with this placement model.

- Successful REPs require careful planning and provision of adequate support mechanisms.

- Development of good practice CSP guidelines would help to facilitate wider use of REPs and educate the profession on their value. 